Hossein Asnaashari / TJMCS Vol. 4 No. 1 (2012) 32 - 36

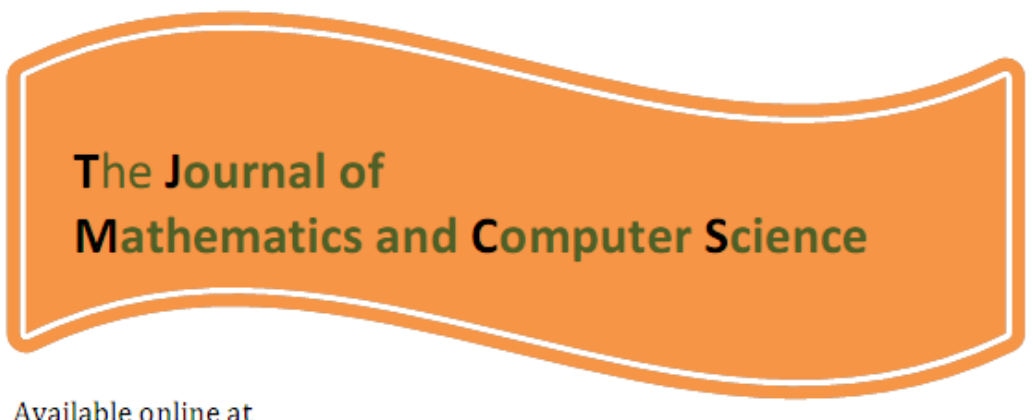

\title{
http://www.TIMCS.com
}

The Journal of Mathematics and Computer Science Vol. 4 No.1 (2012) 32 - 36

\section{TWO CONTINUITY CONCEPTS IN APPROXIMATION THEORY}

\author{
Hossein Asnaashari ${ }^{1}$ \\ Faculty of basic sciences, Zabol University, Zabol, Iran \\ e-mail : h.asnaashar@gmail.com
}

Keywords: Metric projection, best approximation, Chebyshev set, sun, set-valued mapping.

2010 Mathematics Subject Classification: 41A65, 54C60.

Received: December 2011, Revised: March 2012

Online Publication: May 2012

\section{Introduction.}

There has been much recent interest in studying various continuity criteria for the set-valued metric projection onto a set $V$. Particular interest has centered around the relationship between these criteria and either the structure of the set $V$ itself or the geometry of the whole space. See, for example, [3], [4], [7], [8], [10], [16], [17], [18] and [21]. In essentially all of these papers, the concepts of lower semicontinuity (l.s.c.) and/or upper semicontinuity (u.s.c.) for set-valued mappings (as defined, for example, in Hahn [12]) played the key role. In this note we introduce some simpler and more general radial continuity criteria. Roughly speaking, these criteria require that the restriction of the metric projection to certain prescribed line segments be l.s.c. or u.s.c. It turns out that these criteria, which are formally much weaker than l.s.c. or u.s.c, are still strong enough to generalize a number of known results, and weak enough so that many of these theorems now have valid converses (which they did not have under the stronger hypotheses of l.s.c. or u.s.c).

Throughout this note $X$ will denote a (real or complex) normed linear

\footnotetext{
${ }^{1}$ Corresponding author email: h.asnaashar@gmail.com.
} 
space. For $x \in X$ and $r>0$, let

$$
S(x, r)=\{y \in X:\|x-y\|=r\}
$$

The distance from a point $x$ to a subset $V$ of $X$ is defined by

$$
d(x, V)=\inf \{\|x-v\|: v \in V\}
$$

The metric projection onto a subset $V$ of $X$ is the mapping $P_{V}$ which associates with each $x \in X$ its set of best approximations in $V$, i.e.

$$
P_{V}(x)=\{v \in V:\|x-v\|=d(x, V)\}
$$

$V$ is called proximinal (resp. Chebyshev) provided $P_{V}(x)$ contains at least (resp. exactly) one point for each $x \in X$. $V$ is called a sun if for each $x \in X$ and $v \in P_{V}(x), v \in P_{V}(v+\lambda(x-v))$ for every $\lambda \geq 0$. The convex hull of a set $A$ is denoted $\operatorname{co}(A)$. The notation $(x, y)$ is used for the open interval $\{\lambda x+(1-\lambda) y: 0<\lambda<1\}$. $P_{V}$ is said to be l.s.c. (resp. u.s.c.) at $x_{0}$ if, for open set $W$ with $W \bigcap P_{V}\left(x_{0}\right) \neq \emptyset$ (resp. $W \supset P_{V}\left(x_{0}\right)$ ), there exists a neighborhood $U$ of $x_{0}$ such that $W \cap P_{V}(x) \neq \emptyset\left(\right.$ resp. $\left.W \supset P_{V}(x)\right)$ for every $x \in U$.

All other undefined notation or terminology can be found in [11].

\section{ORL continuity.}

The first generalization of 1.s.c. is

Definition 2.1. Let $V \subset X$ and $x_{0} \in X$. $P_{V}$ is said to be outer radially lower (abbreviated ORL) continuous at $x_{0}$ if for each $v_{0} \in P_{V}\left(x_{0}\right)$ and each open set $W$ with $W \cap P_{V}\left(x_{0}\right) \neq \emptyset$, there exists a neighborhood $U$ of $x_{0}$ such that $W \bigcap P_{V}(x) \neq \emptyset$ for every $x$ in $U \bigcap\left\{v_{0}+\lambda\left(x_{0}-v_{0}\right): \lambda \geq 1\right\}$. $P_{V}$ is called ORL continuous if it is ORL continuous at every point.

Theorem 2.2. [14] Let $V \subset X$ and consider the following statements.

(1) $V$ is a sun.

(2) $P_{V}$ is ORL continuous.

(3) For each $x \in X$, every local minimum of the function $\Phi(v)=\|x-v\|$ on $V$ is a global minimum.

(4) $V$ is a "moon" (in the sense of [1]).

Then $(1) \Rightarrow(2) \Rightarrow(3) \Rightarrow(4)$.

The The implication $(3) \Rightarrow(2)$ is false in general (e.g. take $V$ to be the complement of the open unit ball in the Euclidean plane). Also, the implication $(4) \Rightarrow(3)$ is false in general. However, it is an open question whether 
$(2) \Rightarrow(1)$

We call a space $X$ an MS-space if every moon in $X$ is a sun. In such a space all the conditions of Theorem 2.2 are obviously equivalent. In [1], the spaces of type $C(T), T$ compact Hausdorff (or more generally $C_{0}(T)$, $T$ locally compact Hausdorff) and $l_{1}(S)$ for any set $S$, were shown to be MS-spaces. On the negative side, no strictly convex space is a MS-space. In particular, we can state

Corollary 2.3. Let $X$ be an MS-space and $V \subset X$. Then $V$ is a sun if and only if $P_{V}$ is ORL continuous.

It is interesting to compare this result with a particular consequence of two theorems of Vlasov ([21, Theorem 7] and [20, Theorem 13]) which establishes the hard part of the following theorem (cf. also Asplund [2] for an alternate proof):

Theorem 2.4. A Chebyshev set $V$ in a Hilbert space is a sun (i.e. is convex) if and only if $P_{V}$ is continuous.

It is still unknown whether every Chebyshev set in a Hilbert space is convex. In fact, it is apparently unknown whether there exists a Chebyshev set in any space which is not a sun.

\section{ORU continuity.}

A generalization of u.s.c. is given by

Definition 3.1. Let $V \subset X$ and $x_{0} \in X . P_{V}$ is called outer radially upper (abbreviated ORU) continuous at $x_{0}$ if, for each $v_{0} \in P_{V}\left(x_{0}\right)$ and each open set $W \supset P_{V}\left(x_{0}\right)$, there exists a neighborhood $U$ of $x_{0}$ such that $W \supset P_{V}(x)$ for every $x$ in $U \bigcap\left\{v_{0}+\lambda\left(x_{0}-v_{0}\right): \lambda \geq 1\right\}$. $P_{V}$ is called ORU continuous if it is ORU continuous at each point.

Theorem 3.2. [14] Let $V \subset X$ be proximinal and suppose $P_{V}(x)$ is convex for every $x \in X$. If $P_{V}$ is ORU continuous, then $P_{V}(x)$ is compact for every $x \in X$.

This theorem generalizes one of Singer's [18] who had proved it in the particular case when $V$ is a subspace and $P_{V}$ is u.s.c. Theorem 3.2 is false in general if $P_{V}$ is not convex-valued. To see this, we need only take $X=l_{2}$ and $V$ to be the complement of the open unit ball. Then $P_{V}$ is ORU continuous but $P_{V}(0)=S(0,1)$ is not compact.

Theorem 3.3. [14] Let $V$ be a sun such that $P_{V}(x)$ is compact for every $x \in X$. Then $P_{V}$ is ORU continuous.

Combining Theorems 3.2 and 3.3 we obtain

Corollary 3.4. Let $V$ be a proximinal convex set. Then $P_{V}$ is ORU 
continuous if and only if $P_{V}(x)$ is compact for every $x$.

It is worth mentioning that Corollary 3.4 is false with u.s.c. in place of ORU continuity, even if $V$ is a subspace. This follows since there exist Chebyshev subspaces with discontinuous metric projections. (The first such example was given by Lindenstrauss [15, pp. 87-88].)

\section{References}

[1] D. Amir and F. Deutsch, Suns, moons, and quasi-polyhedra, J. Approximation Theory 6 (1972), 176-201.

[2] E. Asplund, Chebyshev sets in Hilbert space, Trans. Amer. Math. Soc. 144 (1969), 236-240.

[3] J. Blatter, Zur Stetigkeit von mengenwertigen metrischen Projektionen, Forschungsber. Landes Nordrhein-westfalen Nr. 1870, Westdeutscher Verlag, Cologne, 1967, pp. 17-38.

[4] J. Blatter, P. D. Morris and D. Wulbert, Continuity of the set-valued metric projection, Math. Ann. 178 (1968), 12-24.

[5] J. Blatter, Weiteste Punkte und nchste Punkte, Rev. Roumaine Math. Pures Appl. 14 (1969), 615-621.

[6] B. Brosowski and R. Wegmann, Charakterisierung bester Approximationen in normierten Vektorrumen, J. Approximation Theory 3 (1970), 369-397.

[7] B. Brosowski and R. Wegmann, On the lower semi-continuity of the set-valued metric projection, J. Approximation Theory.

[8] B. Brosowski, ber eine Fixpunkteigenschaft der metrischen Projektion, Computing 5 (1970), 295-302.

[9] B. Brosowski and F. Deutsch, On some geometrical properties of suns, J. Approximation Theory.

[10] A. L. Brown, Best n-dimensional approximation to sets of functions, Proc. London Math. Soc. (3) 14 (1964), 577-594.

[11] N. Dunford and J. T. Schwartz, Linear operators. I: General theory, Pure and Appl. Math., vol. 7, Interscience, New York, 1958. 
[12] H. Hahn, Reelle Funktionen, Akademie Verlagsgesellschaft, Leipzig, 1932; reprint, Chelsea, New York, 1948.

[13] V. Klee, Remarks on nearest points in normed linear spaces, Proc. Colloq. on Convexity (Copenhagen, 1965), Kobenhavns Univ. Mat. Inst., Copenhagen, 1967, pp. 168-176.

[14] B. Brosowski, F. Deutsch, Some new continuity concepts for metric projections, Bull. Amer. Math. Soc. 78 (6) (1972), 974-978.

[15] J. Lindenstrauss, Extension of compact operators, Mem. Amer. Math. Soc. No. 48 (1964), 112 pp.

[16] P. D. Morris, Metric projections onto subspaces of finite codimension, Duke Math. J. 35 (1968), 799-808.

[17] E. V. Osman, Continuity of metric projection and some geometric properties of the unit sphere in a Banach space, Dokl. Akad. Nauk SSSR 185 (1969), 34-36 = Soviet Math. Dokl. 10 (1969), 291-293.

[18] I. Singer, On set-valued metric projections, Proc. Conf. on Linear Operators and Approximation Theory (Oberwolfach, 1971)

[19] S. B. Steckin, Approximation properties of sets in normed linear spaces, Rev. Math. Pures Appl. 8 (1963), 5-18. (Russian).

[20] L. P. Vlasov, Approximatively convex sets in Banach spaces, Dokl. Akad. Nauk SSSR 163 (1965), 18-21 = Soviet Math. Dokl. 6 (1965), 876-879.

[21] L. P. Vlasov, Chebyshev sets and approximatively convex sets, Mat. Zametki 2 (1967), 191- 200. (Russian). 